\title{
Research of User-Centered Intelligent Technology in China's Cultural and Creative Product Design
}

\author{
CHENG Hui*
}

Information and Technology Department, Zhejiang University of Finance \& Economics Dongfang College, Haining, China

\begin{abstract}
With the development of computer science, "user-centered" design assisted by intelligent technology is a new trend in China's cultural and creative design. To clarify the research progress, the author reviewed the representative predecessor's works in China, summarized a design process paradigm from their works, and classified the various design methods into a reasonable framework by manual content analysis. However, some problems still exist. The first is lack of intelligent design method promotion, and the other is the researchers are unaware that cultural and creative design has developed to a state with blurred boundary, in which the design not only means the design of cultural and creative products but also includes the design of exhibitions and education activities.
\end{abstract}

\section{INTRODUCTION}

"User-centered" is a universal design principle for product. Donald A. Norman divided design features into three levels: visceral design, behavioral design, and reflective design in his Emotional Design. Most of China's cultural and creative product design research is also based on this principle. B. D. Leong from Hong Kong Polytechnic University put forward a three-layer cultural concept, the "spatial perspective of culture", consisting of inner level, middle level, and outer level, which laid a firm foundation. R.T. Lin from Taiwan University of Arts further integrated the above two theories and proposed a basic model of cultural and creative product design. What is more, he also divided the design process into cultural symbols selection, design concept formation and design practice implementation [1]. This hierarchical model (Figure 1) and design process (Figure 2) has almost become the research and practice paradigm of cultural and creative product design in China.

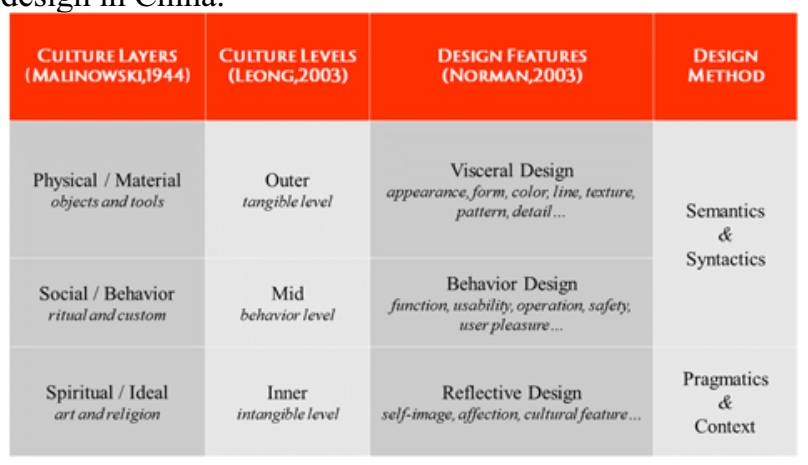

Figure 1. Basic Model of Cultural Product Design.

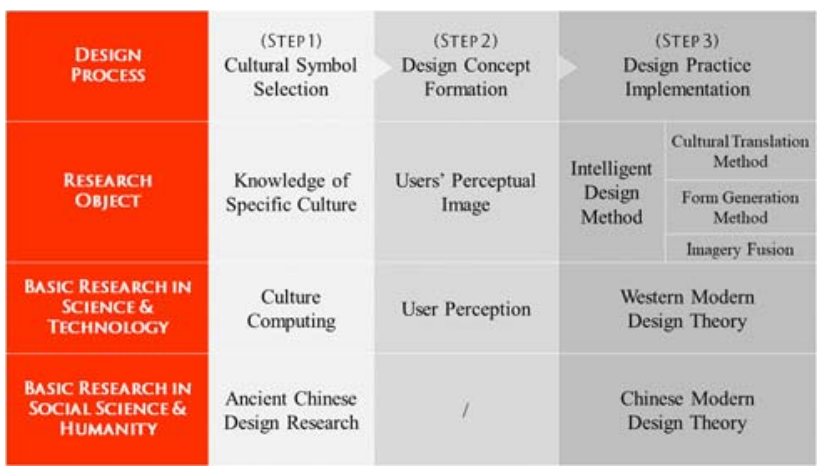

Figure 2. China's Paradigm of Cultrual \& Creative Design.

\section{Cultural Symbols Selection}

"Culturality" is the key to distinguish cultural and creative products from ordinary products. The study of the aesthetics of creation in culture is the basis of cultural and creative product design.

\subsection{Ancient Chinese Design Research}

The research on ancient Chinese design mostly focuses on the analysis of technological background, aesthetic interest, modeling characteristics, symbolic meaning in ancient China. The research is mostly produced in art, history, archeology, anthropology, culture, sociology, design, and other disciplines, such as Zhang Daoyi studied the decorative art of Dunhuang Mogao Grottoes based on the theory of patterns [2]. Han Rong took the pot holding as the research object, and studied the changes in the local form, overall proportion and texture of the pot holding art from the Tang to Song Dynasty [3]. Based on the design concepts and religious beliefs of the Tang Dynasty, Wang

\footnotetext{
*Corresponding author: chenghui2050@163.com
} 
Yali and others analyzed the composition method and pattern meaning of the bronze mirror of the beast pattern and summarized its design characteristics [4].

\subsection{Cultural Computing}

Most research results of traditional cultural relics study are not design knowledge which cannot be used directly in modern design, therefore a "service system" that can make culture elements transformed is needed. In 2005, Tosa Naoko of Kyoto University put forward the concept of "cultural computing", which captures and presents culture in multiple directions through digital technology, combines with analysis of the user's feelings, emotions and subconscious thinking under specific cultural situations and experiences to accurately represent the essence of the culture [5]. One of the current researches in "culture computing" focuses on the field of "metadata", like Zhao Haiying has used the internationally mature DC metadata standard as a reference, combined with the characteristics of clothing patterns, and carried out a metadata description of Miao's butterfly pattern [6]. Wu Yun, another scholar used metadata to digitize ethnic costumes in Xinjiang and explored the cultural connotations and laws of patterns [7].

The original intention of "culture computing" is to establish a knowledge service system to convert specific culture element data to design knowledge. R.T. Lin proposed to use a three-level model to store information of cultural relic [8], in order to translate culture information to design language efficiently. Gou Bingchen also completed the design of the seasoning bottle by constructing a knowledge service system for Banpo painted pottery culture [9].

\section{Design Concept Formation}

The users' perceptual image of cultural and creative products includes two aspects, that is, the image perception at the product modeling level and the user's perception of cultural images.

\subsection{Ways of Acquiring Users' Perceptual Image}

Users' perceptual image research originated from Donald A. Norman's "mental model" research. However, it requires the use of tools to represent it, owing to its complexity.

\subsubsection{Scenario Approach}

As a common method to understand the needs of consumers, the Scenario Approach observes the changes in the users' behaviors in each scene and judges their behavioral purpose and needs in the current scene to discover their true needs. However, this method also has certain drawbacks, that is, the designer's subjective "imagination" may not match the real users' needs, and they may not necessarily recognize it.

\subsubsection{Kansei Engineering}

Compared with the subjective Scenario Approach, Kansei Engineering quantifies perceptual cognition, uses data to characterize the correlation between cultural attributes, modeling parameters and consumer preferences, which can scientifically and accurately identify key elements and get closer to real demand. As a representative method of emotional design, the application of Kansei Engineering in the design of cultural and creative products is quite abundant.

\subsection{Methods of Perceptual Image Analysis}

It is included but not limited to the following four.

\subsubsection{Analytic Hierarchy Process}

The Analytic Hierarchy Process (AHP) is a common method for filtering the importance of perceptual images and is suitable for sorting out ambiguous relationships. Xu Zhanmin applied this method to construct a design factor extraction model for flower-related cultural products, extract design factors from three aspects of form, color, and flower language and calculate the contribution rate of each factor, to obtain a perfect design factor extraction model [10]. Yang Xiaoyan extracted the design elements of the four sacred beasts of the Han Dynasty from the four aspects of form, color, pattern and meaning, carried out semantic analysis, adopted the analytic hierarchy process to choose the above analysis elements, and carried out a flat style transformation in accordance with the needs [11]. Of course, the disadvantage is that it is unable to directly transform users' needs into product design parameters, but to use methods, such as TRIZ to achieve innovative design.

\subsubsection{Factor Analysis}

Factor analysis is mainly used to analyze the potential structure, which can ensure the construction effect of the users' demand evaluation index system to the greatest extent and ensure the accuracy of the model. He Xuemei used factor analysis to extract the styling factors of ladies in the Tang Dynasty [12]. Lu Weijia defined the evaluation factors of Yuelu Academy's culture, selected the form, color, and connotation factors in it, and applied them in product design [13].

\subsubsection{KANO Model}

KANO model can analyze the impact rate of specific needs on customers' satisfaction and can help determine the key perceptual image elements. Chen Junzhi used the KANO model to analyze consumers' traditional cultural innovation design preferences and concluded that "interest" is "unary quality", "individualization" is "necessary quality", and "culturality" is "indifferent quality", which effectively guide the design of cultural dolls [14]. Wang Weiwei used the KANO model to measure the user's response when facing with or without a certain function to determine the functional attribute weight of cultural and 
creative products to assist in the design of cultural and creative products [15].

\subsubsection{Genotyping Method}

Genotyping Method is a method of classifying samples with similar attributes according to rules, which helps to find commonalities among diverse samples. Han Rong constructed the shape map of the ceramic goblet cup in Yuan Dynasty, based on this method, and discussed the relationship between the goblet cup shape and related factors [16]. Zhan Qinchuan used the method to extract the characteristic in the Beginning of Spring and the characteristic in the tea set and used the shape grammar to generate the tea set with the characteristics of the Beginning of Spring [17].

\section{Design Practice Implementation}

Completing the design of cultural and creative products is the process of constructing the mapping relationship between perceptual images and modeling features, as well as the process of transforming requirements into design schemes.

\subsection{Cultural Translation Dimension}

\subsubsection{Pragmatics}

Pragmatics discusses the reasonable cognition of cultural elements in cultural and creative products, including the user's cultural background and thinking mode, and the way in which the product is used. This content is also the basic work when extracting cultural style characteristics, which is mentioned above, so I will not repeat it.

\subsubsection{Context}

Context explores the relationship between cultural and creative products and the environment, including the historical use environment of cultural relics and the modern use environment of products. "Narrative design" is the focus of product context research, and a combination of semiotics and narratives, also known as "story-telling design". There are two modes of narrative design. The one is "birth story", which is based on the birth background of the product, such as culture, history, and cultural relic stories. The other one is "consumption story", which also can be regard as "experience design", through which consumers can experience traditional culture by playing the roles in the story.

\subsubsection{Semantics}

Semantics are divided into explicit semantics and implicit semantics. Explicit semantics focuses on the mapping effects of cultural elements in products' shape, color, material, texture, function, etc. Implicit semantics refers to allegories, that is, abstract parts are conveyed through metaphors and symbols. The more commonly used design rhetoric in the design of cultural and creative products are metaphors and symbols. The first one is metaphor. Based on "similarity" and "association", it discovers the projective relationship between product elements and cultural elements, which can be specifically divided into metaphors, metaphors, metonymy, analogy, and allegory. The second one is symbol. The mapping relations are completed through regulations or conventions, which requires learning to understand, such as a comb symbolizing love. Product designs that focus on design rhetoric are mainly divided into two categories. The one type emphasizes humor and interest, and the other type is bionic design. For example, Lin Hanyu have transformed the explicit shape and implicit meaning of Chinese characters into cultural creative products [18].

\subsubsection{Syntactics}

Syntactics refers to the form of cultural elements on the product and the way of combining them with functional elements, mainly by rotation, repetition, symmetry, gradual change, scaling, specificity, convergence, contrast, rhythm, approximation, dynamics, combination, variation, or other graphic composition rules to generate patterns. Huang Guanghui used the graphic composition rules to design the pattern of the Macau Ruins of St. Paul, forming a tourism cultural and creative product [19]; He Canqun simplified the typical cultural elements of Wuxi and designed a personalized clip with regional characteristics of Wuxi [20].

\subsection{Form Generation Method}

\subsubsection{Shape Grammar}

Shape grammar is a rational method of modeling generation based on computational reasoning. The basic grammar rules, which are similar to graphic composition rules mention above, include but are not limited to addition, deletion, scaling, copying, replacement, rotation, mirroring, displacement, shear and so on (Figure 3). Shape grammar has obvious advantages in pattern generation. For example, Wang Weiwei used shape grammar to derive carpet patterns with Xinjiang characteristics [21]. Zhao Minting used the displacement rules of the shape grammar to recreate the embroidered pomegranate pattern of Qin dynasty [22]. Yang Xiaoyan used the shape grammar to deform and evolve the refined graphic elements of cultural theme of the Spring Festival into a pattern, which was applied to the design of theme poster of the Spring Festival [23]. 


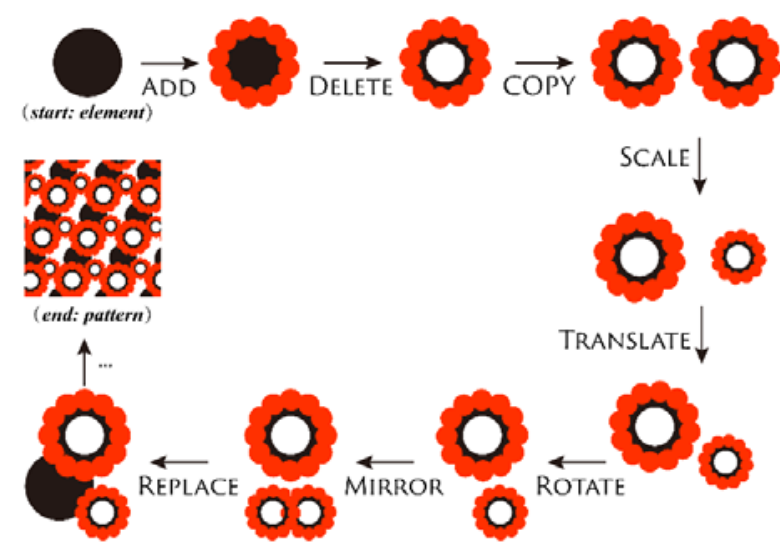

Figure 3. Example of Using Shape Grammar Rules.

\subsubsection{TRIZ Theory}

TRIZ is a product innovation method, which is also applicable to the field of cultural and creative product design. Sun Dongyang proposed a strategy to use the contradiction matrix of TRIZ to solve the problems of cultural and creative products, such as homogeneity, low quality, poor functionality, insufficient practicability, and invariability [24]. Zhou Lili optimized the TRIZ 39*39 contradiction matrix and improved its efficiency in the design of cultural and creative products [25].

\subsection{Imagery Fusion}

\subsubsection{Prototype Theory}

In the design of cultural and creative products, the prototype refers to the typicality of cultural relics in terms of form, color, and decoration, which can inspire consumers and designers. The theory believes that through the analysis and screening of the appearance characteristics of the collection, typical elements can be derived, and because of this, designers can be inspired to construct the matching relationship between cultural relic prototypes and innovative products. In addition, since people's recognition of familiar things is based on the "unconscious behavior" of past experience, the "amplitude" of cultural and creative product design cannot be too large, neither can it be too far away from the prototype of the cultural relic, nor can it be too far from the commonly used product, otherwise it will affect consumers' understanding of cultural and creative products.

\subsubsection{Morphological Matrix Method}

Morphological matrix method decomposes the problem to be solved into several basic elements, lists all the possible means to realize each factor respectively, and then uses the matrix to arrange and combine them, and finally gives the solution. Ge Lu constructed a form innovation matrix from the two dimensions of cultural elements and product carriers and explored the cultural and creative product design of Mongolian folklore [26]. From form, color, pattern and semantics, Deng Li also used matrix thinking, selected Yi's clothing of Liangshan in Sichuan province and combined them with appropriate elements to form a variety of cultural and creative product design schemes [27].

\section{Conclusion}

The research on the design methods of Chinese cultural and creative products has successfully realized the integration of art and technology, and gradually formed a relatively complete methodological system. However, the relevant research has the following limitations. Firstly, academic research is out of touch with industry needs. Some design methods of cultural and creative product are not suitable for commercial design practice, due to cumbersome processes, long design time, and lack of commerciality, unless they can be used to realize intelligent design with the help of computers. Otherwise, it will only be considered "experimental". Secondly, current design focuses on the modeling design itself. "The concept of cultural and creative" has been extended to activities, exhibitions, commodities, and other content [28], which requires design researchers to view the whole industry of cultural and creative from a systematic perspective, and actively introduce the frontier design theories, such as strategic design, service design, organizational design, to adapt to the development of its concept and broaden the research boundary of its products.

\section{ACKNOWLEDGEMENT}

Sponsors: Zhejiang Provincial Philosophy \& Social Science Program (20NDQN322YB), Zhejiang University of Finance \& Economics Dongfang College's Key Research Program (2020dfy003).

\section{REFERENCES}

1. R. T. Lin, "Transforming Taiwan Aboriginal Cultural Features into Modern Product Design: A Case Study of a Cross-cultural Product Design Model," International J. of Design,vol.1,no.2,pp.45-53,2007.

2. D. Y. Zhang, "The Decoration Art of Dunhuang Mogao Grottoes," J. of Nanjing Arts Institute (Fine Arts \& Design),no.2,pp.49-54, 1979.

3. R. Han, "A Comparative Study of Ewer Art in the Tang and Song Dynasties," Art \& Design,no.8,pp.124-125,2008.

4. Y. L. Wang, E. P. Yuan, and F. S. Liu, "Analysis on Design Characteristic of the Deity Animal Button Bronze Mirror in the Tang Dynasty," Packaging Engineering, vol.38,no.20,pp.245-249,2017.

5. H. Y. Zhao, G. Y. Jia, and Z. G. Pan, "Review on the Methods and Applications in Cultural Computing,"Computer Systems \& Applications, vol.25,no.6,pp.1-8,2016. 
6. H. Y. Zhao, G. Y. Jia, and H. Chen, "Construction of Dress Patterns Metadata Oriented Cultural Computation," Progress in Textile Science \& Technology,no.2,pp.31-34,2016.

7. Y. Wu, "The Digital Construction of the Basic Elements of Xinjiang Ethnic Costumes and Applied to the Design," Art \& Design,no.2,pp.85-87,2017.

8. R. T. Lin, R. Cheng, and M. X. Sun, "Digital Archive Database for Cultural Product Design," in International Conference on Usability and Internationalization, 2007,pp.154-163.

9. B. C. Gou, H. Yu, and Z. F. Li, "Study on Gene Extraction and Design Application of Banpo Colored Pottery Culture," J. of North Western Polytechnical University (Social Sciences),vol.31,no.4,pp.6669+104, 2011.

10. Z. M. Xu, and Y. Li, "Application of Extraction Model of Product Design Factors of Floral Culture," J. of Graphics,vol.38,no.1,pp.45-51,2017.

11. X. Y. Yang, S. Liu, and W. W. Wang, "Application of Flat Design Style of Regional Culture Graphic," Packaging Engineering,vol.36,no.20, pp.26-29,2015.

12. X. M. He, and Y. Y. Li, "Form Factors and Design Application of Tang Dynasty Ladies," Packaging Engineering,vol.37,no.12,pp.176-179,2016.

13. W. J. Lu, R. K. He, and D. H. Xiao, "Creative Product Design Based on Yuelu Academy Culture," Packaging Engineering,vol.36,no.24,pp.105$109+114,2015$.

14. J. Z. Chen, and Z. X. Shen, "The Study of Consumer Preference for Traditional Culture Creativity Design: A Case of Figure Designs," J. of National Taiwan College of Arts,vol.89,pp.127-150,2011.

15. W. W. Wang, Y. Z. Liu, and X.Y. Yang, "Cultural and Creative Product Design under User Behavior and Situation Orientation," Packaging Engineering,vol.40,no.24,pp.27-32,2019.

16. R. Han, and W. C. Li, "Genotyping Method to Quantify Patterns and Research of Ceramic Stem Cup of Yuan Dynasty,” Art \& Design,no. 9,pp.74-76,2014.

17. Q. C. Zhan, N. X. Wang, and Z. Y. Li, "Cultural and Creative Tea Set Design and Method Based on the
Beginning of Spring," Packaging Engineering, vol.40,no.8,176-181,2019.

18. H. Y. Lin, R. T. Lin, and H. Y. Xue, "Exploring the Possibilities of Transforming Chinese Characters into Product Design," J. of Design,vol. 10,no.2,pp.77$88,2005$.

19. G. H. Huang, Z. Sun, and Q.Y. Gao, "Application of 'Macau Elements' in the Design of Souvenirs," Art \& Design,no.10,pp.76-77,2011.

20. C. Q. He, J. Li, and X. M. Tang, "Research on Wuxi Personalized Tourism Product Design Based on Cultural Characteristics," Packaging Engineering,vol.37,no.10,118-120,2016.

21. W. W. Wang, X. H. Peng, and X. Y. Yang, "Application Study of Shape Grammar in Evolutionary Design of Traditional Patterns," Packaging Engineering,vol.34,no.6,pp.57-60,2013.

22. M. T. Zhao, and N. Wang, "Qin Embroidery Pomegranate Pattern Visual Elements Extraction and Design Regeneration," Packaging Engineering, vol.39,no.20,pp.8-14,2018.

23. X. Y. Yang, and X. Liu, "Spring Festival Cultural Theme Element Extraction and Derivative Design," Packaging Engineering,vol.40,no.4, pp.93-98,20 19.

24. D. Y. Sun, "Application of TRIZ Theory in the Design of Tourist Souvenirs," Packaging Engineering,vol.38,no.12,pp.248-252,2017.

25. L. L. Zhou, "Optimization and Application of TRIZ Theory in Tourism Souvenir Design," Industrial Design,no.6,pp.98-99,2016.

26. L. Ge, "Innovative Design on Northern China Mongolian Folk Culture Products," Packaging Engineering,vol.40,no.6,pp.46-50,2019.

27. L. Deng, B. Chen, and X. W. Zhang, "The Extraction and Application of Costume Culture Gene of Liangshan Yi Nationality," Packaging Engineering,vol.39,no.2,pp.270-275,2018.

28. H. Cheng, "The Boundary of Museum Cultural Creative and Its Thinking," Newspaper of Chinese Cultural Relics,pp.5,April $14^{\text {th }} 2020$. 